\title{
Feature Extraction Using Rough Set Theory in Service Sector Application from Incremental Perspective
}

\author{
Chun-Che Huang ${ }^{\mathrm{a}}$, Tzu-Liang (Bill) Tseng ${ }^{\mathrm{b}^{*}}$, Chia-Ying Tang ${ }^{\mathrm{a}}$ \\ ${ }^{a}$ Department of Information Management, National Chi Nan University, Taiwan \\ No. 1, University Road, Puli, Nantou 545, Taiwan, ROC \\ cchuang@ncnu.edu.tw; s94213012@ncnu.edu.tw \\ ${ }^{\mathrm{b}}$ Department of Industrial Manufacturing and Systems Engineering, The University of \\ Texas at El Paso \\ 500 West University Avenue, El Paso, TX 79968 \\ btseng@utep.edu
}




\title{
Feature Extraction Using Rough Set Theory in Service Sector Application from Incremental Perspective
}

\begin{abstract}
In service industry application, there is vague and qualitative information required to be processed properly, for example, to identify customer preferences in order to provide adequate services. From literature, Rough Set Theory (RST) has been indicated to be one of promising approaches to cope with vagueness in a large scale database. Basically, the rough set approach integrates learning-from-example techniques, extracts rules from a data set of interest, and discovers data regularities. Most of the existing RS based approaches are able to implement rule induction but it is very time consuming from computation perspective particularly from a large database. To date, there is a demand to generate and analyze business decision rules based on dynamical data sets and conclude such rules on the daily basis in the service industry. Therefore, in this study, an Incremental Weight Incorporated Rule Identification (IWIRI) algorithm is proposed to fulfill such demand. The proposed approach is proficient to efficiently process in-coming data (objetcs) and generate updated decision rules without re-computation efforts in the database. Identification of features based on the customer's preference and implementation of the proposed algorithm are summarized in the case study. This paper forms the basis for solving many other similar problems that occur in service industries.
\end{abstract}

Keywords: Rough Set Theory, Incremental Algorithm, Incremental Object, Rule Induction, Feature Selection, Service Science

\section{Introduction}

Over the past decades, services have become the largest part of most industrialized nations' economies (Spohrer et al., 2007). The explosive growth of services in economies around the world has vast implications for business practice, academic knowledge creation, education, and government policy (Bitner et al., 2008). Service can be defined as the application of competences for the benefit (Lusch and Vargo, 2006), and broadly considered as an application of specialized knowledge, skill, and experience, performed for 
co-creation of respective values of both consumer and provider (Lusch and Vargo, 2006; Qiu et al., 2007; Spohrer et al., 2007).

The real value of a delivered service lies in its ability to satisfy customer's need, which is not simply and strictly shown in the technical characteristics of the service. It is essential to meet customer's needs from a business competitiveness perspective. Particularly, a service significantly affects customer satisfaction. The understanding is also a necessity for a systemic decision-making on how the service system should be transformed for improved customer satisfaction or a competitive advantage (Qiu, 2009).

For the enterprises, to provide services, they must first understand the customer's ideas and preferences. However, with the great number of customers, how do we identify their interests? The answer to this question is to build personalized service (Yu, 1999) and it can be practiced through understanding of customer preference. For example, in aviation service industry, through customer's satisfaction investigation including preference identification relevant to cabin facility, ticket price, safety record, connection service, food and beverage etc., can be conducted to explore particular features when they take the airplanes.

Obviously, feature selection is a core and effective tool for exploring the critical customer features. And the information related to the customer-preferred features is collected through research, interviews, meetings, questionnaires, sampling, and other techniques. These types of data are often discretized and are frequently in "qualitative" format (e.g. salary level, preference level, etc.). Numerous approaches have been applied to feature selection, e.g. generic algorithms (Van Coillie et al., 2007; da Silva et al., 2009; Lee et al., 2006), artificial neural network (ANN) (Saravanan and-Ramachandran, 2010; Chakrabarti, 2008; Glezakos et al., 2009), Tabu Theory (Wang et al., 2010; Wang et al., 2009), branch-and-bound algorithm (Nakariyakul and Casasent, 2007; Chen, 2003), Fuzzy 
C-means Algorithm (Francesco, 2003) and SOM (Laaksonen et al., 2004). However, these approaches are not used for processing qualitative information. They are not suitable for feature selection of customers' preference when enterprise want to service them because the aforementioned methodologies are population-based approaches which may require several statistical assumptions and they have limitations in handling qualitative data (Kusiak, 2001).

One of individual object model-based approaches to deal with qualitative information is the rough set approach (Kusiak, 2001). The rough set theory (RST) is of fundamental importance in artificial intelligence (AI) and cognitive sciences, especially in the areas of machine learning, knowledge acquisition, decision analysis, knowledge discovery from databases, expert systems, decision support systems, inductive reasoning, and pattern recognition (Pawlak, 1991). The rough set approach is suitable for processing qualitative information that is difficult to analyze by standard statistical techniques (Heckerman et al., 1997). It integrates learning-from-example techniques, extracts rules from a data set of interest, and finds data regularities (Komorowski and Zytkow, 1997). To select desired features, the rough set approach attempts to eliminate as many features as possible in the problem domain, and still obtain useful and meaningful outcomes with acceptable accuracy. Having a minimal number of features often leads to establishment of simple models that can be more easily interpreted. Tseng and Huang (2007) proposed the integer-programming methodology and WIRI algorithm to support rule induction more effectively. Moreover, it is also able to handle several conditions, like each feature weight and different objects assigned. Through the weight analysis, the features are highly correlated to customers' characteristics and the preferences can be are identified.

To date, the current rough set based approaches, such as the WIRI algorithm and other non-classic RS-based algorithm, for example Dominance-based Rough Set Approach 
(Greco, Matarazzo and Slowinski, 2001; Greco, Matarazzo and Slowinski, 2002) and its extension Believable Rough Set Approach (Chai and Liu, 2013) are capable to generate a set of decision rules efficiently, but they are not able to generate rules incrementally when new data (i.e., objects) are provided. In practical, the volume of database, big data (Manyika, et al., 2011; Lynch, 2008) is often increased dynamically (Guo et al., 2005). To obtain updated decision rules from the new data set (i.e., the original and additional data), we have to elaborate computation efforts to re-calculate reducts based on the new data set. It should consume significant computation time and memory space, and therefore the efficiency of these algorithms is very low (Liu et al., 2004). Since this is a practical issue in real world, an efficient incremental technique is desired to solve this problem without re-implementing the algorithm in a dynamic database.

From literature, it is apparent that (1) assumptions of static behavior of the database and the drawback that the traditional RS approaches re-implement the algorithm by analyzing the whole database including the incremental data and the original data are frequently observed and (2) inefficiency that the traditional rough set method often induct features with too many rules without focus, and cannot produce rules containing preference order. Therefore, this paper proposes models of the integer-programming and an incremental weight incorporated rule identification (IWIRI) method. The methodology is able to achieve the following objectives:

- Search the minimal number of feature rules for decision marking when the new data is add-in without re-compute the entire data set.

- Aggregate the weight of the feature and the frequency of the object to search for the optimal rules under the situation when new objects are given.

- Identify outcomes and significant features simultaneously.

- Validate the superiority of the proposed approach by a case study related to the 
aviation domain. In this case study, the IWIRI algorithm is used and allows managers handle and classify the new customers' data more efficient.

The remainder of this paper is organized as follows: Section 2 presents the integer-programming models to the incremental rough set-based rule induction problem due to provision of new objects. Particularly, confliction and converge associated with the original rules are incorporated to various solution cases. In Section 3, the WIRI algorithm and the IWIRI algorithm are developed to resolve limitation of the IP models. A case study in Section 4 is illustrated application of market analysis of the airline customer through the use of the proposed methodology. Section 5 concludes the paper.

\section{The Incremental Rough Set-based Rule Induction Problem}

Given by a set of data and their associated reducts, this section models the incremental problem when a new set of data are added in. Through implementing the exhaustive search procedure, five types of results are classified:

Case I The object's increment does not cause any conflict with original rules, and the original rules can cover the new object.

Case II The new object's increment does not cause any conflict with original rules, but the original rules cannot cover this new object.

Case III Increment of the new object causes conflict with the original rules, but it can be covered by one of the original reducts/rules.

Case IV Increment of the new object causes conflict with original rules, and the new object cannot be covered by one of the original reducts.

Case V Increment of the new object causes conflict with the original rule, and new object's features are identical with one of the original objects, but the two objects have different output. 
Note that "conflict" refers to that the new object has same feature as the original rules, but the new objects has different output with the original rules. "The original rule covers the new object" refers to that the new object fits to the original rules in the condition (i.e., premise) part.

In order to formulate the incremental- integer programming model, the following notations are used:

$$
\begin{aligned}
& i \text { : index of reduct } \\
& j: \text { index of feature } \\
& p: \text { index of object } \\
& n: \text { number of features } \\
& m: \text { number of reducts }
\end{aligned}
$$$$
R_{p} \text { : set of reducts obtained from object } p \text {, }
$$$$
\mathrm{A}=\left[a_{i j}\right]_{m \times n} \text { : A reduct-feature incidence matrix which refers to the set of arcs }
$$
connecting reducts from set $R_{p}$ to set $R_{q},(p, q) \in P \times P$ and $p \neq q$

$$
a_{i j}=\left\{\begin{aligned}
e_{i j}, & \text { if reduct } i \text { includes feature } j \\
x, & \text { reduct } i \text { and feature } j \text { has not any relationship }
\end{aligned}\right.
$$$$
x_{i}= \begin{cases}1, & \text { if reduct } i \text { is selected } \\ 0, & \text { otherwise }\end{cases}
$$$$
y_{i j}= \begin{cases}1, & \text { if reduct } i \text { and reduct } j \text { have been selected } \\ 0, & \text { otherwise }\end{cases}
$$

$d_{i j}$ : Distance measure between reduct $i$ and $j$, where $d_{i j}=\sum_{\mathrm{k}=1}^{\mathrm{n}-1} \delta\left(a_{i k}, a_{j k}\right)$

$\delta\left(a_{i k}, a_{j k}\right)= \begin{cases}0, & \text { if } a_{i k}=a_{j k}=" \mathrm{x} " \\ 1, & \text { otherwise }\end{cases}$

$P:$ is the set of reducts $\{1,2, \ldots, l\}$ to be selected, where $\left|\cup_{p=1}^{l} R_{p}\right|=m$

$R_{p}=$ the set of reducts for object number $p$, where $\cup R_{p}=m, p \in P$

$m_{0} \quad=$ number of original objects' reducts

$m_{\text {new }} \quad=$ number of new objects' reducts

$m_{\text {conflicted }}=$ reduct number of original objects' reduct that conflicts with the new object

Corresponding to each case, a LP model is presented. The model is assumed that a unique final rule is selected from the reducts according to an object in the induction process. Therefore, the objective function desires to minimize the total distance between any two 
reducts $i$ and $j$ from the same object since searching the similar/identical reducts from other objects is an ultimate goal for reduct selection.

\section{$\underline{\text { Case I }}$}

Case I refers to when the new object increment does not cause any conflict with original rules and the original rules can cover any new object. That is, the original rules are not changed by this new increment object. But if the new object has more than one original rules and can cover the new object, then choose the rule which has smaller distance with the new object to cover the new object. The final output rules are same as the original rules.

\section{Integer-programming module of Case I}

$\min \frac{1}{2} \sum_{(i, j) \in A} d_{i j} y_{i j}$

subject to: $\quad \sum_{i \in R p} x_{i}=1, p \in P$

$x_{i}+x_{j}-1 \leq y_{i j},(i, j) \in A ; i \neq j$

$\sum_{j \notin R p} d_{i j} x_{i}-\min \left\{\sum_{j \notin R p} d_{k j}\right\} x_{k} \leq 0$, for $k \in R_{p}$,

for $i \neq k, i \in R_{p}$,

for all $i=1, \ldots, m_{0}$

for all $j=1, \ldots, m_{0}$

$y_{i j} \geq 0,(i, j) \in A ; i \neq j$

Constraint (1) ensures that for each object exactly one reduct is selected. Consistency is imposed by constraint (2). Constraint (3) ensures that the reduct used similar feature is selected. Constraint (4) ensures integrality for all features and reducts from original objects.

\section{Case II}

New object increment does not cause any conflict with original rules, and the original rules cannot cover this new object. The algorithm only computes the reducts of 
the new object and the distance of the new object's reducts. And the reduct that has the minimum distance should be the final rule of the new object.

\section{Integer-programming module of Case II}

$\min \frac{1}{2} \sum_{(i, j) \in A} d_{i j} y_{i j}$

subject to: $\quad \sum_{i \in R p} x_{i}=1, p \in P$

$x_{i}+x_{j}-1 \leq y_{i j},(i, j) \in A ; i \neq j$

$\sum_{j \notin R p} d_{i j} x_{i}-\min \left\{\sum_{j \notin R p} d_{k j}\right\} x_{k} \leq 0$, for $k \in R_{p}$,

for $i \neq k, i \in R_{p}$,

for all $i=m_{0+1}, \ldots, m_{0+} m_{\text {new }}$

for all $j=1, \ldots, m_{0}+m_{\text {new }}$

$y_{i j} \geq 0,(i, j) \in A ; i \neq j$

Constraints (5), (6), and (7) are similar in case I. Constraint (8) ensures integrality for all features and reducts from original and new objects.

\section{Case III}

New object increment causes conflict with original rules, but it can be covered by one of the original reducts or rules. In this case, the new object changes the rule which the reduct can cover the new object, and the rule can cover the new object. The algorithm re-computes the reducts of those objects in which the rules conflict with the new object. After computing the new object's reduct, compute these reducts' distance to find the minimum one as the final rule for these objects. Then merge the same rules together and update them to the final rules.

\section{Integer-programming module of Case III}

$\min \frac{1}{2} \sum_{(i, j) \in A} d_{i j} y_{i j}$

subject to: $\quad \sum_{i \in R p} x_{i}=1, p \in P$

$x_{i}+x_{j}-1 \leq y_{i j},(i, j) \in A ; i \neq j$

$\sum_{j \notin R p} d_{i j} x_{i}-\min \left\{\sum_{j \notin R p} d_{k j}\right\} x_{k} \leq 0$, for $k \in R_{p}$,

for $i \neq k, i \in R_{p}$,

for all $i=m_{\text {conflicted }}$

for all $j=1, \ldots, m_{0}+m_{\text {new }}$ 
$y_{i j} \geq 0,(i, j) \in A ; i \neq j$

Constraints (9), (10), and (11) are similar in case I. Constraint (12) ensures integrality for all features in the original and new objects and reducts from the conflicted objects.

\section{$\underline{\text { Case IV }}$}

The new object's incremental causes conflict with original rules, and the new object cannot be covered by one of the original reducts. The original objects' reduct which conflicts with the new object and the new object's reducts are re-compute by the algorithm. And find the minimum distance of these reducts for the objects as the final rule, update the new rules.

\section{Integer-programming module of Case IV}

$\min \frac{1}{2} \sum_{(i, j) \in A} d_{i j} y_{i j}$

subject to:

$\sum_{i \in R p} x_{i}=1, p \in P$

$x_{i}+x_{j}-1 \leq y_{i j},(i, j) \in A ; i \neq j$

$\sum_{j \notin R p} d_{i j} x_{i}-\min \left\{\sum_{j \notin R p} d_{k j}\right\} x_{k} \leq 0$, for $k \in R_{p}$,

for $i \neq k, i \in R_{p}$,

for all $i=m_{0+1}, \ldots, m_{0+} m_{\text {new }}, m_{\text {conflicted }}$

for all $j=m_{0+1}, \ldots, m_{0+} m_{\text {new }}, m_{\text {conflicted }}$

$y_{i j} \geq 0,(i, j) \in A ; i \neq j$

Constraints (13), (14), and (15) are similar in case I. Constraint (16) ensures integrality for all features and reducts from the all objects, including original, new , and conflicted.

\section{$\underline{\text { Case V }}$}

Increment of the new object causes conflict with the original rule, and new object's features are identical with one of the original objects, but the two objects have different outputs. In this case, the objects (new objects and the object which has same features but 
different outputs with the new object) should be deleted. For example, in Table 1, the new object is added in the last row.

Table 1. The incremental object in Case V

\begin{tabular}{|l|l|l|l|l||l|}
\hline Object No. & $\mathbf{F}_{\mathbf{1}}$ & $\mathbf{F}_{\mathbf{2}}$ & $\mathbf{F}_{\mathbf{3}}$ & $\mathbf{F}_{\mathbf{4}}$ & Output \\
\hline $\mathbf{1}$ & 0 & 2 & 1 & 2 & 2 \\
\hline $\mathbf{2}$ & 1 & 2 & 1 & 0 & 2 \\
\hline $\mathbf{3}$ & 1 & 0 & 2 & 0 & 1 \\
\hline $\mathbf{4}$ & 0 & 1 & 2 & 1 & 0 \\
\hline $\mathbf{5}$ & 0 & 1 & 2 & 1 & 1 \\
\hline
\end{tabular}

\section{Integer-programming model of Case V}

$\min \frac{1}{2} \sum_{(i, j) \in A} d_{i j} y_{i j}$

subject to: $\quad \sum_{i \in R p} x_{i}=1, p \in P$

$x_{i}+x_{j}-1 \leq y_{i j},(i, j) \in A ; i \neq j$

$\sum_{j \notin R p} d_{i j} x_{i}-\min \left\{\sum_{j \notin R p} d_{k j}\right\} x_{k} \leq 0$, for $k \in R_{p}$,

for $i \neq k, i \in R_{p}$,

for all $i=1, \ldots, m_{0}-m_{\text {conflicted }}$

for all $j=1, \ldots, m_{0}-m_{\text {conflicted }}$

$y_{i j} \geq 0,(i, j) \in A ; i \neq j$

Constraints (17), (18), and (19) are similar in case I. Constraint (20) ensures integrality for all features and reducts from the original objects, but excluding conflicted objects.

Solving the model for case VI results the following final solution:

$y_{15}=y_{16}=y_{17}=y_{18}=1, y_{25}=y_{26}=y_{27}=y_{28}=1, y_{35}=y_{36}=y_{37}=y_{38}=1, y_{45}=y_{46}=y_{47}=y_{48}=1$,

$y_{57}=y_{58}=1, y_{67}=y_{68}=1, x_{2}=1, x_{5}=1, x_{7}=1$

The integer-programming model is able to generate optimal solutions for a given case.

But modeling an LP or ILP is a time-consuming task and requires Operation Research experts to compute the model, which is not practical for most companies. The integer-programming model assumes that equal weight is assigned to each feature, which is unreasonable in the real-world case. In order to solve large-scale reduct selection 
problems, provide flexibility of weight assignment, and reduce the burden of developing sophisticated mathematical models, an efficient heuristic is very beneficial and is presented next.

\section{Solution Approach}

The proposed algorithm inducts rules based on two previous studies: First, it produces reducts based on the reduct generation procedure from Pawlak (1991), where a reduct is defined as a minimal sufficient subset of a set of attributes which has the same ability to discern concepts as when the full set of attributes is used (Pawlak, 1991, Ziarko, 1994). Since there may be more than one reduct corresponding to an object, in the second part, decision rules are inducted from the reducts using the WIRI algorithm (Tseng and Huang, 2007). Corresponding to each object, meaningful and unique decision rules are derived from the reducts obtained from the first part. This proposed approach focuses on the incremental issues and therefore, skips the details of reduct generation and introduction to basic concepts of rough set theory. The essence of the WIRI algorithm is presented next.

\subsection{The Weight Incorporated Rule Identification (WIRI) algorithm}

In this algorithm, the weight of each feature assumes different from each other due to real world practice. A more comprehensive case when features and objects are of unequal importance, and each of them carries a weight, will be considered here. For example, one object may be assigned a higher weight than another one because of the frequency each appears. In addition, some features might be more important than others and therefore be assigned a higher weight.

In Table 2, the weight value of the feature is subjected to positive integer which is less than or equal to 1 . It can be obtained from domain experts' judgment or through implementing the Analytic Hierarchy Process (AHP) approach. The incidence matrix A = 
$\left[a_{i j}\right]_{m \times n}$, frequency $f_{i}$ for object $i, i=1, \ldots, m$ and weight $w_{j}$ for input feature $j, j=1, \ldots, n$ are associated. It is possible to assign frequency $f_{i}, i=1, \ldots, m$ since the frequency of each object can be derived from the original database. Furthermore, it is also possible to assign weight $w_{j}, j=1, \ldots, n$ since the weight of each feature can be determined from domain experts. Table 1 is expanded with a column indicating the number of objects and a row containing weights associated with the features. Using the weight coefficients $w_{j}$ and $f_{i}$, an auxiliary matrix $\left[e_{i j}\right]$ will be generated from the original reduct - input feature matrix.

Table 2. Data set with un-equal weight for object and feature

\begin{tabular}{|l|l|l|l|l||l||l||l|}
\hline Object No. & $\mathrm{F}_{1}$ & $\mathrm{~F}_{2}$ & $\mathrm{~F}_{3}$ & $\mathrm{~F}_{4}$ & Output & $\begin{array}{l}\text { Object } \\
\text { cardinality }\end{array}$ & $\begin{array}{l}\text { Weight } \\
f_{i}\end{array}$ \\
\hline 1 & 0 & 2 & 1 & 2 & 2 & 4 \\
\hline 2 & 1 & 2 & 1 & 0 & 2 & 3 & 0.75 \\
\hline 3 & 1 & 0 & 2 & 0 & 1 & 2 & 0.5 \\
\hline 4 & 0 & 1 & 2 & 1 & 0 & 3 & 0.75 \\
\hline Weight $w_{j}$ & 0.8 & 0.9 & 1 & 0.7 & \multicolumn{1}{|c|}{} \\
\hline
\end{tabular}

It is clear that equal weight for each feature and object forms as a special situation of un-equal weight case. Therefore, the proposed Weight Incorporated Rule Identification (WIRI) algorithm is capable to handle both cases.

The entries of the transformed entries are defined as follows:

$$
e_{i j}=f_{i \times}\left(w_{j \times} v_{j}\right)
$$

where:

$$
\begin{aligned}
& e_{i j}=\text { entry of the transformed reduct-input feature matrix } \\
& f_{i}=\text { weight of reduct } i \\
& w_{j}=\text { weight of feature } j \\
& v_{j}=1 \text { if feature } j \neq \text { "x"; } 0 \text { otherwise }
\end{aligned}
$$

\section{The WIRI algorithm}

Step 0.

(i) List the auxiliary matrix.

(ii) Compare the reducts (rows of matrix $\left[a_{i j}\right]$ ). Select the features used from only a single reduct of the object(s).

(iii) List the number of known value for each column in $\left[a_{i j}\right]$. Select the potential features used, based on the higher number of known value (refer the results 
from (ii)).

(iv) Set iteration number $k=1$.

Step 1. Compare those reducts (rows of matrix $\left[a_{i j}\right]^{(\mathrm{k})}$ ) for one specific case at a time. Select the reducts from the potential features used and based on the auxiliary matrix. If more than one solution for the reduct selection then select the reduct which can be merged by most of objects; otherwise, select the reducts which are most frequently selected from previous iterations. Draw a horizontal line hi through each row of matrix $\left[a_{i j}\right]^{(\mathrm{k})}$ corresponding to these reducts.

Step 2. For each column in $\left[a_{i j}\right]^{(\mathrm{k})}$ corresponding to an entry of feature which is not " $x$ ", single crossed by any of the horizontal lines hi, draw a vertical line $v_{j}$.

Step 3. Repeat step 1 and step 2 until one reduct has been selected for each object in current outcome. All double-crossed entries of features of the matrix form the rules.

Step 4. If all of objects have been concerned in current outcome, transform the incidence $\operatorname{matrix}\left[a_{i j}\right]^{(\mathrm{k})}$ into $\left[a_{i j}\right]^{(\mathrm{k}+1)}$ by removing all the rows and columns included in current outcome.

Step 5. If matrix $\left[a_{i j}\right](\mathrm{k}+1)=$ " “ (where " " denotes a matrix with all elements equal to blank, stop and output the results; otherwise set $k=k+1$ and go to step 1 .

\subsection{The incremental rule induction for each case}

After an object is added in, the 5 types of results ( 5 cases) are classified (please refer to Section 2). In this section the solution approach corresponding to each case is presented in Figure 1. 


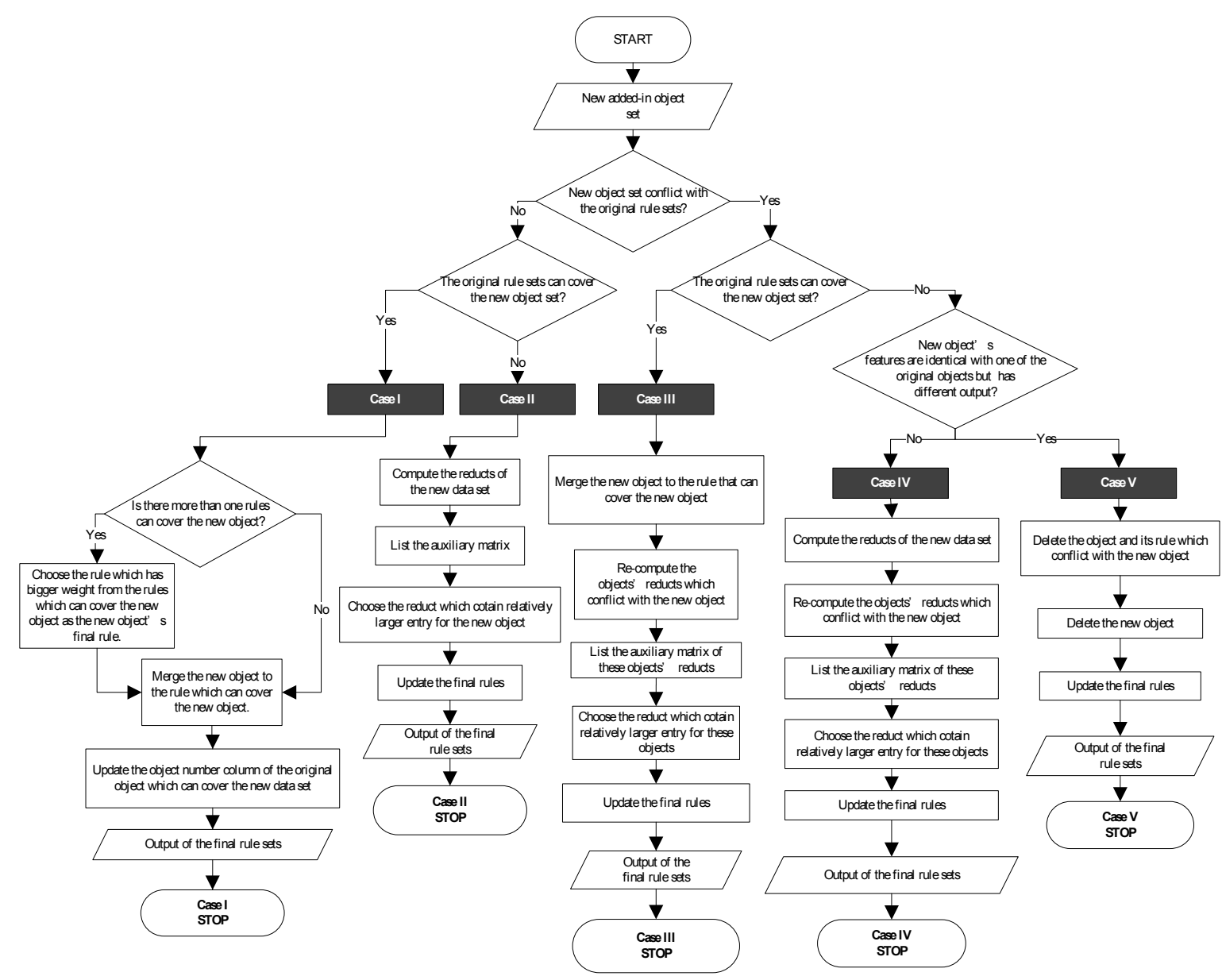

Figure 1. The flow diagram shows the procedure of the proposed incremental algorithm

Five examples are presented to illustrate the fives cases. The raw data is presented in Table 2. The rules inducted by the WIRI approach are illustrated: IF $\left(F_{3}=1\right)$ THEN (Output $=2)$, IF $\left(F_{2}=0\right)$ THEN (Output $\left.=1\right)$, and IF $\left(F_{2}=1\right)$ THEN (Output $\left.=0\right)$. The new objects are added corresponding to each case in Table 3.

Table 3. The incremental objects

\begin{tabular}{|l|l|l|l|l|l||l|l|l|}
\hline & Object No. & $\mathbf{F}_{\mathbf{1}}$ & $\mathbf{F}_{\mathbf{2}}$ & $\mathbf{F}_{\mathbf{3}}$ & $\mathbf{F}_{\mathbf{4}}$ & Output & Object cardinality & Weight $\boldsymbol{f}_{\boldsymbol{i}}$ \\
\hline Example 1 & 5 & 1 & 2 & 1 & 1 & 2 & 1 & 0.25 \\
\hline Example 2 & 6 & 0 & 2 & 2 & 0 & 2 & 1 & 0.25 \\
\hline Example 3 & 7 & 2 & 1 & 1 & 2 & 0 & 1 & 0.25 \\
\hline Example 4 & 8 & 2 & 0 & 0 & 1 & 0 & 1 & 0.25 \\
\hline Example 5 & 9 & 0 & 2 & 1 & 2 & 1 & 1 & 0.25 \\
\hline
\end{tabular}

\section{Example 1}

The new object (object 5 in Table 3 ) can be covered by one original rule. The algorithm does not need to compute reducts to induct the rules, only merge the new 
object's object number to the column of the Object No. This example refers case I: When the new object increment does not cause any conflict with original rules, and the original rules can cover any new object. Then the original rules do not change by this new increment object. The final output rules are same as the original rules. But when new object has more than one original rules can cover the new object, than choose the rule which has bigger weight with the new object to cover the new object. The final result is presented in Table 4.

Table 4. The final solution of Example 1

\begin{tabular}{|l|l|l|l|l||l|}
\hline Object No. & $\mathbf{F}_{\mathbf{1}}$ & $\mathbf{F}_{\mathbf{2}}$ & $\mathbf{F}_{\mathbf{3}}$ & $\mathbf{F}_{\mathbf{4}}$ & Output \\
\hline $\mathbf{1 , 2} \mathbf{5}$ & $\mathrm{x}$ & $\mathbf{x}$ & $\mathbf{1}$ & $\mathrm{x}$ & 2 \\
\hline $\mathbf{3}$ & $\mathrm{x}$ & $\mathbf{0}$ & $\mathbf{x}$ & $\mathrm{x}$ & 1 \\
\hline $\mathbf{4}$ & $\mathrm{x}$ & $\mathbf{1}$ & $\mathbf{x}$ & $\mathrm{x}$ & 0 \\
\hline
\end{tabular}

\section{Example 2}

In this example, the new object (object 6 in Table 3) do not has any conflict with the original rules and cannot be covered by the original rules. The algorithm has only need to compute the new object's reduct set and list the auxiliary matrix to choose the reduct that has the relatively larger value of weight as the new rule.

Step 0 .

(i) The reduct matrix and the auxiliary matrix are shown in Figure 2 and Figure 3.

(ii) Set iteration number $k=1$.

Step 1.Select the reduct based on the potential features used and the auxiliary matrix. Since reduct $8[\mathrm{x} 2 \times \mathrm{x}$ 2] of object 5 is the only single reduct of the object, feature 2 is selected. One horizontal line $h l$ is drawn in Figure 2.

Step 2.One vertical line $v 1$ is drawn in Figure 2.

Step 3.Since the new object contains its selected reduct in current outcome, output the new reduct and the final rules show in Table 5.

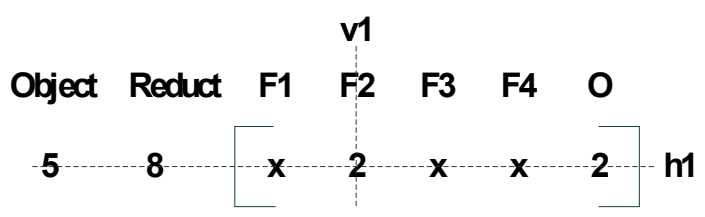

Figure 2. Matrix 1 


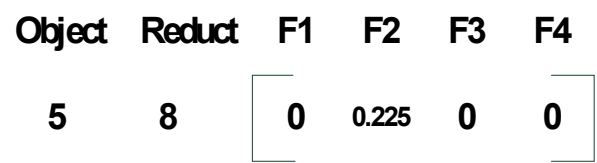

Figure 3. Matrix 2

Table 5. The final solution of Example 2

\begin{tabular}{|l|l|l|l|l||l|}
\hline Object No. & $\mathbf{F}_{\mathbf{1}}$ & $\mathbf{F}_{\mathbf{2}}$ & $\mathbf{F}_{\mathbf{3}}$ & $\mathbf{F}_{\mathbf{4}}$ & Output \\
\hline $\mathbf{1 , 2}$ & $\mathrm{x}$ & $\mathbf{x}$ & $\mathbf{1}$ & $\mathrm{x}$ & 2 \\
\hline $\mathbf{3}$ & $\mathrm{x}$ & $\mathbf{0}$ & $\mathbf{x}$ & $\mathrm{x}$ & 1 \\
\hline $\mathbf{4}$ & $\mathrm{x}$ & $\mathbf{1}$ & $\mathbf{x}$ & $\mathrm{x}$ & 0 \\
\hline $\mathbf{5}$ & $\mathrm{x}$ & $\mathbf{2}$ & $\mathbf{x}$ & $\mathrm{x}$ & 2 \\
\hline
\end{tabular}

This example refers to Case II: When the new object's incremental does not cause any conflict with the original rules, and the original rules cannot cover this new object. The algorithm computes the reducts of the new object, and list the auxiliary matrix for these reducts. Choose the reduct which has the larger entry or by the potential feature and which can be merged by most objects, then output the new rule of the new object.

\section{Example 3}

In this example, the new object (object 7 in Table 3) has conflict with the original rules but can be covered by the other original rule. The algorithm need to compute-the objects' reducts which conflict with the new objects, than list the auxiliary matrix to choose the reduct that has the relatively larger value of weight as the changes rules. Step 0 .

The reduct matrix and the auxiliary matrix are shown in Figure 4 and Figure 5.

(i) Set iteration number $k=1$.

Step 1.Select the reduct based on the potential features used and the auxiliary matrix. Since reduct $1[\mathrm{x} 2 \mathrm{x} \times 2$ ] of object 1 and reduct $2[\mathrm{x} 2 \mathrm{x} \times 2]$ of object 2 is the only single reduct of these two objects, feature 2 is selected. Two horizontal lines $h l$ and $h 2$ are drawn in Figure 4.

Step 2.One vertical line $v l$ is drawn in Figure 4.

Step 3.Since the new object contains its selected reduct in current outcome, output the new reducts and the final rules show in Table 6. 


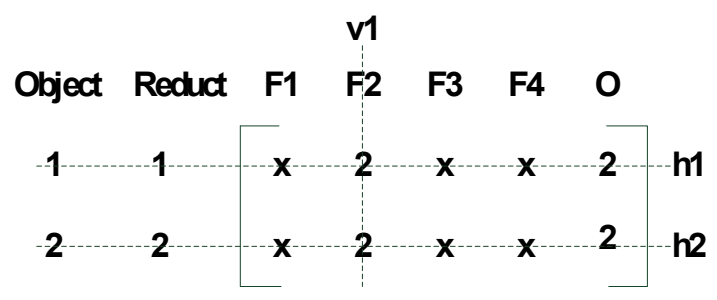

Figure 4. Matrix 3

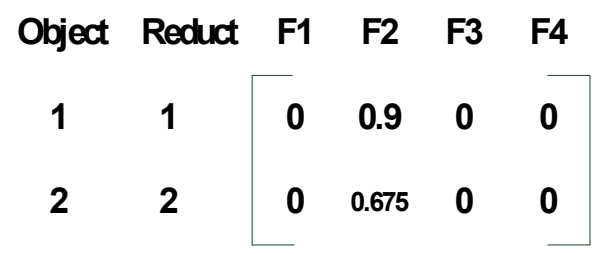

Figure 5. Matrix 4

Table 6. The final solution of Example 3

\begin{tabular}{|l|l|l|l|l||l|}
\hline Object No. & $\mathbf{F}_{\mathbf{1}}$ & $\mathbf{F}_{\mathbf{2}}$ & $\mathbf{F}_{\mathbf{3}}$ & $\mathbf{F}_{\mathbf{4}}$ & Output \\
\hline $\mathbf{1 , 2}$ & $\mathbf{x}$ & $\mathbf{2}$ & $\mathbf{x}$ & $\mathbf{x}$ & $\mathbf{2}$ \\
\hline $\mathbf{3}$ & $\mathrm{x}$ & 0 & $\mathrm{x}$ & $\mathrm{x}$ & 1 \\
\hline $\mathbf{4 , 5}$ & $\mathbf{x}$ & $\mathbf{1}$ & $\mathbf{x}$ & $\mathbf{x}$ & $\mathbf{0}$ \\
\hline
\end{tabular}

This example refers to Case III: The new object increment causes conflict with original rules, but it can be covered by other object's rule. So the new object can be merged into the rule which can cover the new object, and the original objects which generate the rules that conflict with the new object's reducts are calculated. And list the auxiliary matrix for these reducts. Choose the reduct which has the larger entry or by the potential feature and which can be merged by most objects, then-output the new rule.

\section{Example 4}

In this example, the new object (object 8 in Table 3) has conflict with the original rules and cannot be covered by the original rules. The algorithm need to compute the new object's reduct set and the original objects' reducts which conflict with the new object. Then list the auxiliary matrix to choose the reduct that has the relatively larger value of weight as the new rules.

Step 0.

(i) The reduct matrix and the auxiliary matrix are shown in Figure 6 and Figure 7.

(ii) Set iteration number $k=1$. 
Step 1.Select the reduct based on the potential features used and the auxiliary matrix.

Since reduct 9 contain the relatively larger entries for object 3 . One horizontal lines $h l$ is drawn in Figure 6.

Step 2.Two vertical line $v 1$ and $v 2$ are drawn in Figure 6.

Step 3.Since each object contains its' selected reduct in current outcome, so go to step 4.

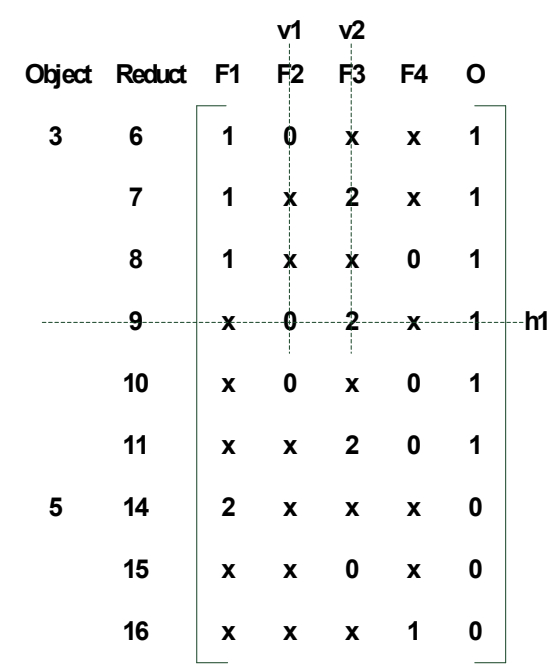

Figure 6. Matrix 5

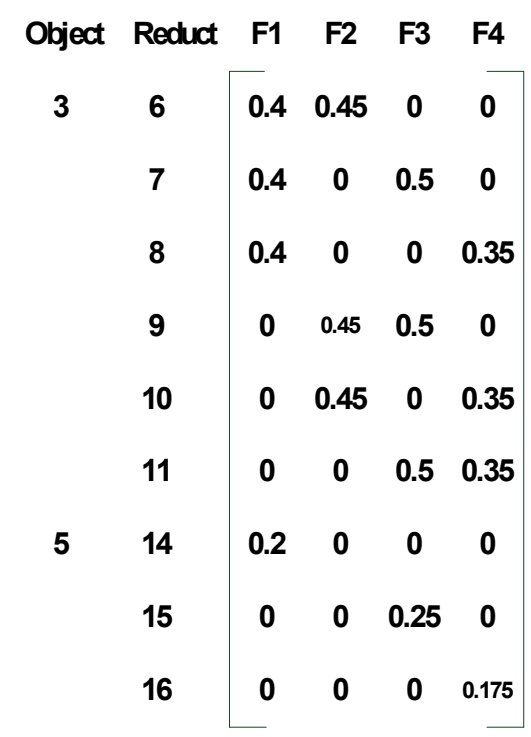

Figure 7. Matrix 6

Step 4.Transform the incidence matrix $\left[a_{i j}\right]^{(1)}$ into $\left[a_{i j}\right]^{(2)}$ by removing all the rows and columns included in outcome $=1$.

Step 5.Set $k=k+1=2$ and go to Step 1 .

Step 1.Select the reduct based on the potential features used and the auxiliary matrix.

Since reduct 15 contain the relatively larger entries for object 5. One horizontal lines $h 2$ is drawn in Figure 8. 
Step 2.One vertical line $v 3$ is drawn in Figure 8 and the updated auxiliary matrix is shown in Figure 9.

Step 3.Since the new object contains its selected reduct in current outcome, output the new reducts and the final rules show in Table 7.

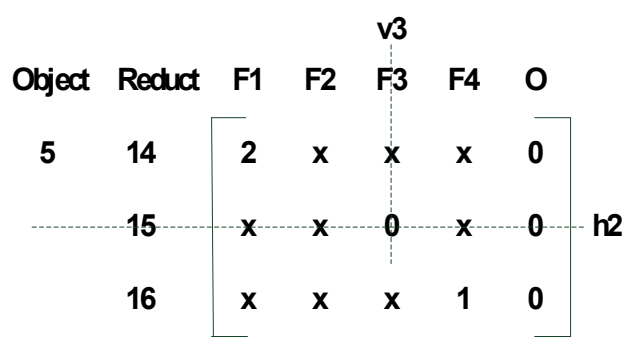

Figure 8. Matrix 7

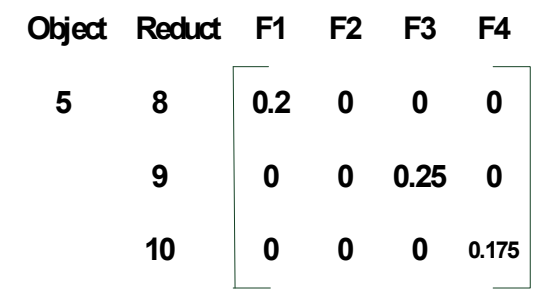

Figure 9. Matrix 8

Table 7. The final solution of Example 4

\begin{tabular}{|l|l|l|l|l||l|}
\hline Object No. & $\mathbf{F}_{\mathbf{1}}$ & $\mathbf{F}_{\mathbf{2}}$ & $\mathbf{F}_{\mathbf{3}}$ & $\mathbf{F}_{4}$ & Output \\
\hline $\mathbf{1 , 2}$ & $\mathrm{x}$ & $\mathrm{x}$ & 1 & $\mathrm{x}$ & 2 \\
\hline $\mathbf{4}$ & $\mathrm{x}$ & 1 & $\mathrm{x}$ & $\mathrm{x}$ & 0 \\
\hline $\mathbf{5}$ & $\mathrm{x}$ & $\mathbf{x}$ & $\mathbf{0}$ & $\mathbf{x}$ & $\mathbf{0}$ \\
\hline $\mathbf{3}$ & $\mathrm{x}$ & $\mathbf{0}$ & $\mathbf{2}$ & $\mathbf{x}$ & $\mathbf{1}$ \\
\hline
\end{tabular}

This example refers to Case IV: New object increment causes conflict with original rules, and cannot be covered by any other original rules. This case is similar to case III, but in this case the new object's rule are not be merged by other rule from original objects.

\section{Example 5}

In this example, the new object (object 9 in Table 3) has conflict with the original rules and cannot be covered by the original rules. Also the new object has identical features with the conflict original rule's object. The algorithm has no need to re-compute the object's reduct, the process is to delete the new object and the original rule which conflict with the new object. The final result is presented in Table 8 . 
Table 8. The final solution of Example 5

\begin{tabular}{|l|l|l|l|l||l|}
\hline Object No. & $\mathbf{F}_{\mathbf{1}}$ & $\mathbf{F}_{\mathbf{2}}$ & $\mathbf{F}_{\mathbf{3}}$ & $\mathbf{F}_{\mathbf{4}}$ & Output \\
\hline $\mathbf{2}$ & $\mathrm{X}$ & $\mathrm{X}$ & 1 & $\mathrm{X}$ & 2 \\
\hline $\mathbf{3}$ & $\mathrm{x}$ & 0 & $\mathrm{x}$ & $\mathrm{X}$ & 1 \\
\hline $\mathbf{4}$ & $\mathrm{x}$ & 1 & $\mathrm{X}$ & $\mathrm{X}$ & 0 \\
\hline
\end{tabular}

This example refers to Case V: New object conflicts with the original rules, and the new object's features are identical with one of the original object's features but the two objects have different output. The objects (new objects and the object which has same features but different output with the new object) are deleted.

\subsection{The time complexity of the proposed incremental algorithm}

Based on the 5 cases in Section 3.2, the algorithm can solve the problems of data add-in but no need to re-compute the entire data from the very beginning. When given a set of data to add into the original data set, the algorithm add the new data one by one via a loop and then revises the original rules. By this approach, the method proposed in this paper can not only solve the incremental problems but also decrease the time complexity. The time complexity of the IWIRI and the original WIRI algorithms is presented in Table 9, which shown the IWIRI has less complexity than WIRI.

Table 9. Time complexity of this proposed algorithm is composed of five cases

\begin{tabular}{|l|l|l|}
\hline Method & Description & $\begin{array}{l}\text { Time complexity in the } \\
\text { worst case }\end{array}$ \\
\hline $\begin{array}{l}\text { The IWIRI } \\
\text { algorithm }\end{array}$ & $\begin{array}{l}\text { It is capable to handle the new add-in data by } \\
\text { partially re-computing. }\end{array}$ & $\mathrm{O}(m(p n)+m(r n))$ \\
\hline $\begin{array}{l}\text { The original } \\
\text { WIRI } \\
\text { algorithm }\end{array}$ & $\begin{array}{l}\text { It is NOT capable to handle the in-coming data } \\
\text { individually except re-computing the entire } \\
\text { updated data set (i.e., the original plus additional } \\
\text { data) }\end{array}$ & $\mathrm{O}(m p(p n+q n))$ \\
\hline
\end{tabular}

$p$ : denotes the total number of the original objects,

$q$ : denotes the total number of the new objects,

$r$ : denotes the total number of object which generated the rule (or reduct) that conflict with the new data set,

$m$ : denotes the number of the original reducts,

$n$ : denotes the total number of attributes

\section{Case Study}


The continental ABC hotel group which represents the largest entrepreneurship in mainland China, plans to develop its strategic plan for the different customer groups. The market manager wants to recognize customers' satisfaction and opinions through customers' profiles. To know the customers' preference and apply different marketing strategies, the customer-preferred features of the airline should be discovered. The proposed approach is applied to find out the core features.

In this case, 14 related features, including the demographic information about the customers and other criterion that VIP customers may consider, are present in Table 10. Feature 1 illustrates the customer's gender. Feature 2 depicts customer's age that is classified as different levels. Feature 3 represents the career of the customer, including government employee, employee of company, student and others. Feature 4 represent the income of the customers', this feature can show their economic situation. Feature 5 represents if customers feel convenient and efficient when they make a reservation and check in. Feature 6 represents customers' concern with the facility on the plane, including cleanliness of interior and seats; seating comfort; seat space and leg room. Feature 7 represents the service attitude and professional of the flight attendant that customers feel. Feature 8 represents the entertainment facility on the plane, including audio and video system, games, and variety of newspaper and magazine. Feature 9 represents the promptness, accuracy of baggage delivery and the probability of luggage lose and damage that customer concerned, and the follow-up reimbursement when lose and damage occur. Feature 10 represents the previous safety and reliability data about the airline. Feature 11 represents the status of on time departure and arrival. Feature 12 represents customers' concern with the price of the ticket. Feature 13 represents the quality of the food and beverage that customers feel. Feature 14 represents the prompt handling of customer's requests and complaints for the unexpected cases, for example, 
flight delay or cancellation. Feature 15 represents the flight time configuration in the airline (Chang and Yehg (2002); Gilbert and Robin Wong (2003); Liou (2009); Liou and Tzeng (2010); Liou (2011)). The output feature O represents customer satisfaction level. A partial data set of 10 customers in this case study is illustrated in Table 11, and the weight of each features are presented in Table 12.

Table 10. Customer-preferred features of the airlines

\begin{tabular}{|c|c|c|c|c|c|}
\hline & Attribute name & 0 & 1 & 2 & 3 \\
\hline F1 & Gender & Female & Male & -------- & --------- \\
\hline $\mathrm{F} 2$ & Age & Less than 30 & $30-40$ & $\begin{array}{ll}41 & \text { and } \\
\text { above }\end{array}$ & -------- \\
\hline F3 & Occupation & $\begin{array}{l}\text { Government } \\
\text { employee }\end{array}$ & $\begin{array}{l}\text { Employee of } \\
\text { company }\end{array}$ & Student & Others \\
\hline F4 & Income & $\begin{array}{ll}\text { Less } & \text { than } \\
30,000 & \end{array}$ & $30,000-70,000$ & $\begin{array}{l}70,000 \text { and } \\
\text { above }\end{array}$ & -------- \\
\hline F5 & Booking and check-in service & Low & Medium & High & -------- \\
\hline F6 & Cabin facility & Low & Medium & High & -------- \\
\hline F7 & In-flight service & Low & Medium & High & -------- \\
\hline F8 & In-flight entertainment & Low & Medium & High & -------- \\
\hline F9 & Luggage Delivery Service & Low & Medium & High & --------- \\
\hline F10 & Safety record & Low & Medium & High & --------- \\
\hline F11 & $\begin{array}{l}\text { Status of on-time } \\
\text { departure/arrival }\end{array}$ & Low & Medium & High & -------- \\
\hline F12 & Price & Low & Medium & High & -------- \\
\hline F13 & Food and beverage & Low & Medium & High & ------- \\
\hline F14 & Complaints handling & Low & Medium & High & --------- \\
\hline F15 & Timetables & Low & Medium & High & --------- \\
\hline $\mathrm{O}$ & Customers' satisfaction & Low & Medium & High & --------- \\
\hline
\end{tabular}

Table 11. A typical data set for 10 types of customer

\begin{tabular}{|l|l|l|l|l|l|l|l|l|l|l|l|l|l|l|l|l|}
\hline & F1 & F2 & F3 & F4 & F5 & F6 & F7 & F8 & F9 & F10 & F11 & F12 & F13 & F14 & F15 & O \\
\hline 1 & 0 & 1 & 0 & 1 & 1 & 1 & 1 & 2 & 1 & 2 & 1 & 1 & 1 & 2 & 1 & 2 \\
\hline 2 & 0 & 1 & 3 & 0 & 1 & 2 & 2 & 2 & 2 & 2 & 2 & 0 & 1 & 1 & 2 & 2 \\
\hline 3 & 1 & 0 & 1 & 2 & 1 & 1 & 1 & 0 & 1 & 2 & 0 & 2 & 1 & 2 & 0 & 1 \\
\hline 4 & 0 & 0 & 1 & 1 & 1 & 1 & 2 & 1 & 2 & 1 & 1 & 1 & 0 & 1 & 2 & 1 \\
\hline 5 & 1 & 2 & 0 & 1 & 2 & 2 & 2 & 1 & 1 & 2 & 1 & 1 & 2 & 1 & 2 & 2 \\
\hline 6 & 0 & 2 & 1 & 1 & 1 & 2 & 2 & 1 & 1 & 2 & 2 & 1 & 1 & 1 & 1 & 1 \\
\hline 7 & 0 & 0 & 2 & 0 & 1 & 2 & 2 & 0 & 1 & 1 & 1 & 0 & 0 & 1 & 2 & 1 \\
\hline 8 & 1 & 2 & 0 & 2 & 1 & 1 & 2 & 2 & 2 & 2 & 2 & 2 & 1 & 1 & 1 & 2 \\
\hline 9 & 1 & 2 & 3 & 1 & 2 & 1 & 2 & 2 & 2 & 2 & 2 & 1 & 1 & 2 & 2 & 2 \\
\hline 10 & 1 & 1 & 0 & 1 & 1 & 1 & 2 & 1 & 2 & 1 & 2 & 1 & 1 & 1 & 1 & 1 \\
\hline
\end{tabular}

Table 12. Weight table for each feature

\begin{tabular}{|l|c|c|c|c|c|c|c|c|c|c|c|c|c|c|c|}
\hline Feature & F1 & F2 & F3 & F4 & F5 & F6 & F7 & F8 & F9 & F10 & F11 & F12 & F13 & F14 & F15 \\
\hline Original value & 60 & 65 & 70 & 75 & 75 & 70 & 75 & 60 & 75 & 95 & 90 & 90 & 60 & 75 & 80 \\
\hline Ratio(weight) & 6 & 6.5 & 7 & 7.5 & 7.5 & 7 & 7.5 & 6 & 7.5 & 9.5 & 9 & 9 & 6 & 7.5 & 8 \\
\hline $\begin{array}{c}\text { Normalized } \\
\text { weight }\end{array}$ & 0.63 & 0.68 & 0.78 & 0.79 & 0.79 & 0.78 & 0.79 & 0.63 & 0.79 & 1 & 0.95 & 0.95 & 0.63 & 0.79 & 0.89 \\
\hline
\end{tabular}




\subsection{Computational results}

The historical data are split into two data sets based on the ratio: 0.632 for the training set and 0.368 for the test set (Kusiak, 2001). In this case study a $7 \%$ threshold value is determined based on a marketing specialist's expertise for the final reduct sets, which are presented in Table 13.

Table 13. The reduct sets compute from the original data by the WIRI algorithm

\begin{tabular}{|c|c|c|c|c|c|c|c|c|c|c|c|c|c|c|c|c|}
\hline & $\overline{\mathrm{F} 1}$ & $\overline{\mathrm{F} 2}$ & $\overline{\mathrm{F} 3}$ & $\overline{\mathrm{F} 4}$ & F5 & $\overline{\mathrm{F} 6}$ & $\overline{\mathrm{F} 7}$ & $\overline{\mathrm{F} 8}$ & F9 & $\overline{F 10}$ & $\overline{F 11}$ & F12 & F13 & $\overline{\mathrm{F} 14}$ & F15 & $\mathrm{O}$ \\
\hline 1,8 & $\mathrm{X}$ & $\mathrm{X}$ & $\mathrm{X}$ & $\mathrm{X}$ & X & $\mathrm{X}$ & $\mathrm{X}$ & 2 & $\mathrm{X}$ & X & X & $\mathrm{X}$ & X & $\mathrm{X}$ & X & 2 \\
\hline 2 & $\mathrm{X}$ & $\mathrm{X}$ & 3 & $\mathrm{X}$ & $\mathrm{X}$ & $\mathrm{X}$ & $\mathrm{X}$ & $\mathrm{X}$ & $\mathrm{X}$ & X & $\mathrm{X}$ & X & $\mathrm{X}$ & $\mathrm{X}$ & $\mathrm{X}$ & 2 \\
\hline $5,9,11,12$ & X & $\mathrm{X}$ & $\mathrm{X}$ & $\mathrm{X}$ & 2 & $\mathrm{X}$ & $\mathrm{X}$ & $\mathrm{X}$ & X & X & $\mathrm{X}$ & $\mathrm{X}$ & $\mathrm{X}$ & $\mathrm{X}$ & X & 2 \\
\hline 3,7 & $\mathrm{X}$ & $\mathrm{X}$ & $\mathrm{X}$ & $\mathrm{X}$ & $\mathrm{X}$ & $\mathrm{X}$ & $\mathrm{X}$ & 0 & $\mathrm{X}$ & $\mathrm{X}$ & $\mathrm{X}$ & $\mathrm{X}$ & $\mathrm{X}$ & $\mathrm{X}$ & $\mathrm{X}$ & 1 \\
\hline 4,15 & $\mathrm{X}$ & $\mathrm{X}$ & $\mathrm{X}$ & $\mathrm{X}$ & $\mathrm{X}$ & $\mathrm{X}$ & $\mathrm{X}$ & $\mathrm{X}$ & X & $\mathrm{X}$ & $\mathrm{X}$ & $\mathrm{X}$ & 0 & $\mathrm{X}$ & X & 1 \\
\hline 14,16 & $\mathrm{X}$ & $\mathrm{X}$ & $\mathrm{X}$ & $\mathrm{X}$ & $\mathrm{X}$ & $\mathrm{X}$ & $\mathrm{X}$ & $\mathrm{X}$ & $\mathrm{X}$ & $\mathrm{X}$ & $\mathrm{X}$ & $\mathrm{X}$ & $\mathrm{X}$ & 0 & $\mathrm{X}$ & 0 \\
\hline 6 & $\mathrm{X}$ & $\bar{X}$ & 1 & $\bar{X}$ & $\bar{X}$ & $\bar{X}$ & $\bar{X}$ & $\mathrm{X}$ & $\bar{X}$ & 2 & $\bar{X}$ & $\bar{X}$ & $\bar{X}$ & $\mathrm{X}$ & $\bar{X}$ & 1 \\
\hline 10 & $\mathrm{X}$ & $\mathrm{X}$ & $\mathrm{X}$ & $\mathrm{X}$ & $\mathrm{X}$ & $\mathrm{X}$ & $\mathrm{X}$ & $\mathrm{X}$ & X & 1 & 2 & $\mathrm{X}$ & $\mathrm{X}$ & $\mathrm{X}$ & $\mathrm{X}$ & 1 \\
\hline 13 & $\mathrm{X}$ & $\mathrm{X}$ & $\mathrm{X}$ & $\mathrm{X}$ & $\mathrm{X}$ & $\mathrm{X}$ & $\mathrm{X}$ & $\mathrm{X}$ & $\mathrm{X}$ & 1 & $\mathrm{X}$ & 2 & $\mathrm{X}$ & $\mathrm{X}$ & $\mathrm{X}$ & 1 \\
\hline
\end{tabular}

(I) IF (F8 = 2) THEN (Customers' satisfactory= “2”) [2, 13\%]

(II) $\quad$ IF (F3= 3) THEN (Customers' satisfactory= “2”) [1, 6\%]

(III) IF (F5 = 2) THEN (Customers' satisfactory= “2”) [4, 25\%]

(IV) IF (F8 = 0) THEN (Customers' satisfactory = "1") [2, 13\%]

(V) $\quad$ IF (F13 = 0) THEN (Customers' satisfactory= "1") [2, 13\%]

(VI) IF (F3 = 1) AND (F10 = 2) THEN (Customers' satisfactory = "1") [1, 6\%],

(VII) IF (F10 = 1) AND (F11 = 2) THEN (Customers" satisfactory = “1”) [1, 6\%],

(VIII) IF (F10 = 1) AND (F11 =2) THEN (Customers' satisfactory = “1”) [1, 6\%],

(IX) $\quad$ IF (F14 = 0) THEN (Customers" satisfactory = “0”) [2, 13\%]

For example, the first rule (I) shows - If "the degree of in-flight entertainment is high" Then “customers' satisfactory is high" leading to $13 \%$ of population support such rule.

Through new data collection over time, new VIP customers' data are involved and requires seeing if there is any impact on existing rules. Due to limitations and constraints of the current budget, it is very in-efficient to re-implement the program by taking the 
entire data to get the final decision rules. The IWIRI algorithm is applied next to deal with the problem of new added- in objects' problems.

Table 14 shows the first new object of the new VIP customer's data should add in to get the new decision rules.

Table 14. The first new add-in data

\begin{tabular}{|l|l|l|l|l|l|l|l|l|l|l|l|l|l|l|l|}
\hline F1 & F2 & F3 & F4 & F5 & F6 & F7 & F8 & F9 & F10 & F11 & F12 & F13 & F14 & F15 & O \\
\hline 0 & 0 & 2 & 0 & 1 & 1 & 1 & 1 & 1 & 1 & 1 & 1 & 1 & 1 & 2 & 1 \\
\hline
\end{tabular}

In Table 14, the new object $\left[\begin{array}{llllllllllllllll}0 & 0 & 2 & 0 & 1 & 1 & 1 & 1 & 1 & 1 & 1 & 1 & 1 & 1 & 2 & 1\end{array}\right]$ does not make any conflict with the existing rules, and cannot covered by any of the original rules. Thus this new object can be classified as case II. This case needs to compute the reducts of the new object set, and list the auxiliary matrix for these reducts. Then choose the reduct which has the larger entry or can be merged by most objects, then output the new rule calculated from the new object. Compare to the original rules, there is one new rule that generate by the new add in object: IF $(\mathrm{F} 10=1)$ AND $(\mathrm{F} 15=2)$ THEN (Customers' satisfactory $=1)$

Table 15 shows the second new object of the new VIP customer's data that add in to get the new decision rules.

Table 15. The second new add-in data

\begin{tabular}{|l|l|l|l|l|l|l|l|l|l|l|l|l|l|l|l|}
\hline F1 & F2 & F3 & F4 & F5 & F6 & F7 & F8 & F9 & F10 & F11 & F12 & F13 & F14 & F15 & O \\
\hline 1 & 1 & 1 & 2 & 1 & 1 & 2 & 2 & 1 & 1 & 1 & 1 & 1 & 2 & 2 & 1 \\
\hline
\end{tabular}

In Table 15, the new object [ $\left.\begin{array}{llllllllllllllll}1 & 1 & 1 & 2 & 1 & 1 & 2 & 2 & 1 & 1 & 1 & 1 & 1 & 2 & 2 & 1\end{array}\right]$ has conflict with the rule (I) 'IF (F8 = 2) THEN (Customers' satisfactory=2)". The new object cannot be covered by other original rules. Therefore, this new object can be classified into case IV. In this case, the new object and the objects which generate the rule that conflict with the new object's reduct should be re-compute and list the auxiliary matrix. Apply the algorithm, the final output rules produces three new rules:

Rule 1: IF (F7 = 1) AND (F12 = 1) THEN (Customers' satisfactory = 2)

Rule 2: IF (F11 = 2) AND (F12 = 2) THEN (Customers' satisfactory = 2) 


\footnotetext{
Rule 3: IF (F10 = 1) AND (F11 = 1) THEN (Customers' satisfactory = 1) and the original rule IF $(\mathbf{F 8}=\mathbf{2})$ THEN (Customers' satisfactory=2) that was generated by the conflict object 1 and object 8 has been deleted.
}

\subsection{Discussion}

The objective of this case study is to extract the rules to describe the relationship among traveler's profile and airline services (i.e., conditional features) and customer's satisfaction (i.e., decision feature). The end results focus on the use of rules to classify the background and preferences of the traveler corresponding to their satisfaction levels. Consequently, the findings should help the tourist service agencies develop a couple of competitive travel packages. For example, based on the newly derived rule, the "Medium" rate of in-flight service and price should carry out "High" customer satisfaction. Therefore, the Chief Executive Officer (CEO) should apply this decision rule to (1) propose an in-flight service enhancement plan to train steward and stewardess, and (2) launch a promotion package to appeal more customers.

Per the second derived rule, the administrator should realize that "on-time departure and arrival" and the sale price are critical to customer satisfaction. Consequently, the airline company should take actions to (1) secure the pilots are always on time in their duties and the aircraft is well maintained and (2) provide a airfare discount to appeal more customers.

According to the third rule, "Safety record" and "Status of on-time departure/arrival" are important to customer's satisfaction. It is suggested that the company should (1) maintain "excellent" safety record through the enhancive pilot training program and the "zero breakdown" aircraft program and (2) maintain "on-time" record through educating pilots, stewards and stewardesses to the goal of "no late flight." 
This solution approach also provides the case study for the company when there are in-coming data (i.e., a VIP customer) which require to be used and generate updated solutions in order to establish new marketing strategies. The first new object does not cause any conflict with the original rules but also cannot be covered by the original rules. Hence, the new object does not make any changes to the original rules, and establishes a new rule to the result. This implies that the nature of market does not change even the new high profile object is collected and analyzed. The second new added-in data has conflicted with the original rules and cannot be covered by other original rules. This makes the original rules which conflict with the new object changed. And the new object creates a new rule for the end result. It implies that the nature of market has been changed due to this VIP object involving in this market. The market strategy may require further amendment.

Next, investigation of less computation effort through the proposed approach is conducted. The CPU time of the original WIRI and the proposed IWIRI algorithm are compared. It shows that there are almost $100 \%$ time saving of reduct induction and rule induction can be saved in Case I, $69.7 \%$ time saving of reduct induction and $90.2 \%$ of rule induction time saving in Case II, $85.1 \%$ time saving of reduct induction and $77.8 \%$ time saving of rule induction in Case IV. The proposed approach is less time-consuming than re-computing the entire data set including the new data in these three cases. It is especially useful in extracting rules from large databases since the algorithm do not have to re-compute the entire reducts or rules for the new added-in data. 
Table 16. Summary of experiments based on different cases

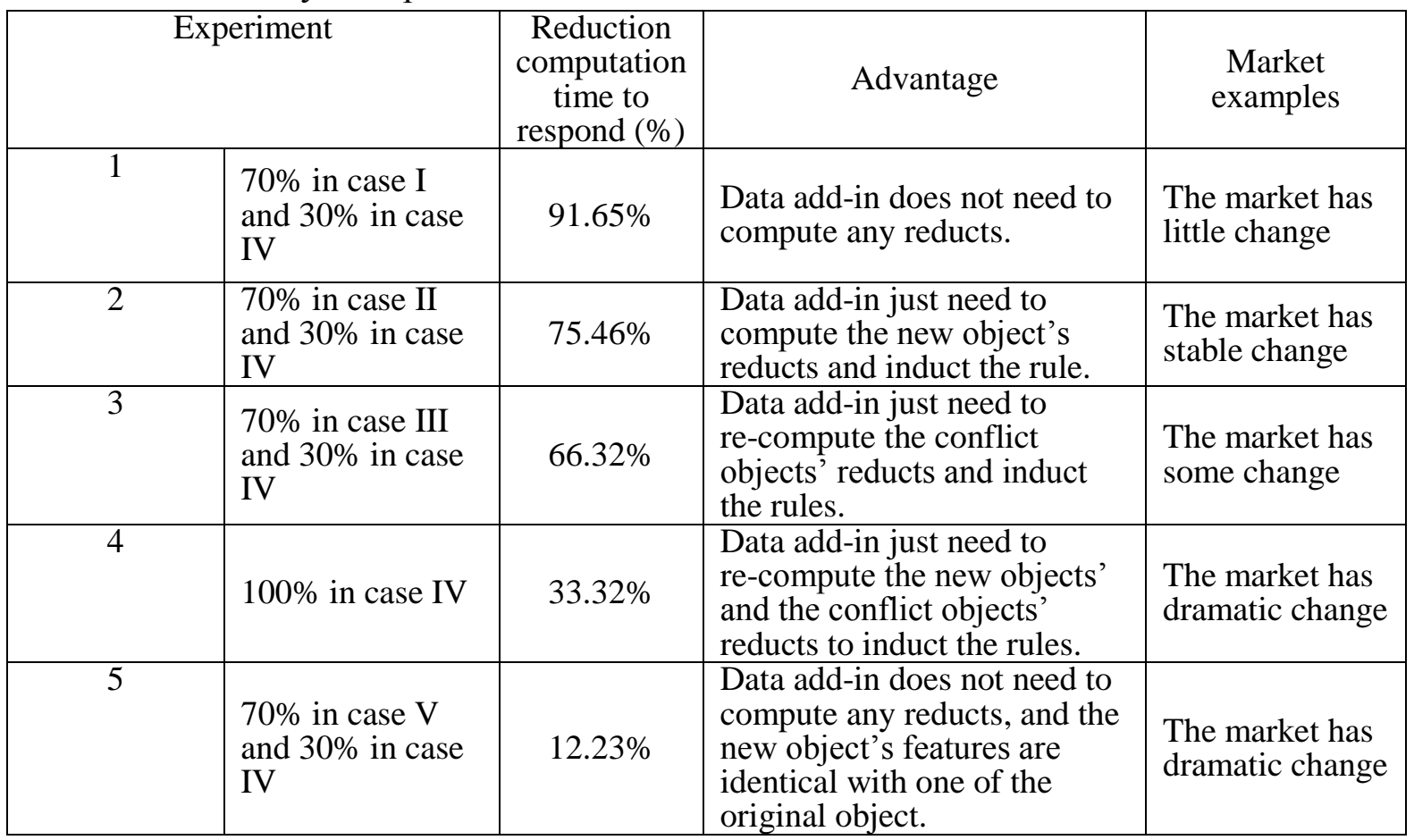

\section{Conclusion}

In this study, the related literatures of traditional RS approaches and incremental technique were reviewed and then the drawbacks of them in previous literatures were presented. The incremental rule-extraction algorithm IWIRI was proposed based on the WIRI of Tseng and Huang (2007) to solve the problems of new objects added-in to the database, and it is unnecessary to re-compute the whole database again. Examples for the cases to understand how the approach searches for solutions were illustrated. At last, a case was studied in the domain of the aviation to validate the proposed approach. This paper aims the decision maker to solve the incremental issues with the following contribution:

- When a new object is add in to the database, it is unnecessary to re-compute the entire object sets for rule sets from the very beginning, the proposed approach can update rule sets by partly modifying original rule sets, and a lot of times are saved. 
- The proposed approach is especially useful in extracting rules from large databases since it does not need to deal with the original rules which do not influenced by the add-in objects. This is an advantage what the traditional incremental rough set based approaches are insufficient.

- The validation of the proposed algorithm is presented in Section 4-2 for each case, from the results can clearly show that the IWIRI algorithm reduce the waste of time and bring effects and benefits to the problem of incremental.

Further studies are required as follows:

- In a dynamic database, the decisions may consider to add-in the new features or data and features deleted-out. However, this study only considers the problems of objects' incremental problem, the domain of data deleted-out problems, feature incremental and feature delete-out problems are not considered in this research. Therefore, it is required to develop a solution for these problems.

- The current algorithm requires the data table to be complete, for example, without missing values about the condition attributes. However, the data tables are very often incomplete (Greco, 2001) in real life. Therefore, it is necessary to consider this situation when the new add-in objects have missing value in the condition features.

- The weight of each feature in the cases is determined by domain experts' judgment and external assessment in the measurement industry, but the experts' opinion is too subjective for the measurement industry. Therefore, using Analytic Hierarchy Process (AHP) or sensitivity methodology to assess effects of weight of the features and identify the most appropriate value is necessary.

- The algorithm proposed in this research mainly uses the WIRI algorithm, but the basic concept is to observe the difference set between the reducts/rules after incremental objects are added in and the original reducts/rules. The differences are 
classified as five cases. Corresponding to different cases, new add-in objects are observing the difference between the original rules to resolve the incremental problem. Finally the new sets of rules are inducted without re-computing the entire data set. Moreover, the algorithm also applies the concept of weight to select rules by opinions of decision makers and domain experts. The concept is not limited to use the WIRI and it can be extended to any different methodologies utilized Rough Set Theory, for example, like Dominance-based Rough Set Approach (Greco, Matarazzo and Slowinski, 2001; Greco, Matarazzo and Slowinski, 2002) and its extension Believable Rough Set Approach (Chai and Liu, 2013)

- When and how often the user has to check the new added-in data and apply the incremental algorithm to update the original rules for the correctly response toward the market variation is a topic should be considered.

\section{References}

Chai, J. and Liu, J. N., 2013, “A Novel Believable Rough Set Approach for Supplier Selection," to appear in Expert Systems with Applications.

Chakrabarti, S., 2008, "Voltage stability monitoring by artificial neural network using a regression-based feature selection method," Expert Systems with Applications, Vol.35, pp.1802-1808

Chang, Yu-Hern and Yehg, Chung-Hsing, 2002, “A survey analysis of service quality for domestic airlines," European Journal of Operational Research, Vol. 139, pp. 166-177

Chen, Xue-wen, 2003, "An improved branch and bound algorithm for feature selection," Pattern Recognition Letters, Vol.24, pp. 1925-1933

da Silva, S.F., Traina, A., Ribeiro, M.X., do E.S. Batista Neto, J. and Traina, C., 2009, "Ranking Evaluation Functions to Improve Genetic Feature Selection in Content-Based Image Retrieval of Mammograms," Computer-Based Medical Systems 22nd IEEE International Symposium on , pp. 1

Francesco M., 2003, "Feature selection based on a modified fuzzy c-means algorithm with supervision,” Information Sciences, Vol.151, pp. 201-26 
Gilbert, David and Wong, Robin K.C., 2003, "Passenger expectations and airline services: a Hong Kong based study," Tourism Management, Vol. 24, pp. 519-532.

Glezakos, Thomas J., Tsiligiridis, Theodore A., Iliadis, Lazaros S., Yialouris, Constantine P., Maris, Fotis P. and Ferentinos, Konstantinos P., 2009, "Feature extraction for time-series data: An artificial neural network evolutionary training model for the management of mountainous watersheds," Neurocomputing, Vol.73, pp.49-59

Greco, S., Matarazzo, B. and Slowinski, R., 2002, "Rough sets methodology for sorting problems in presence of multiple attributes and criteria," European journal of operational research, Vol.138(2), pp. 247-259.

Greco, Salvatore, Matarazzo, Benedetto and Slowinski, Roman, 2001, "Rough sets theory for multicriteria decision analysis," European Journal of Operational Research, vol. 129, No. 1, pp.1-47

Guo, Sen, Wang, Zhi-Yan, Wu, Zhi-Cheng and Yan, He-Ping, 2005, "A novel dynamic incremental rules extraction algorithm based on rough set theory," In Proceedings of the fourth international conference on machine learning and cybernetics, Guangzhou, China, pp.18-21.

Heckerman, D., Mannila, H., Pregibon, D. and Uthurusamy, R., 1997, Proceedings of the third International Conference on Knowledge Discovery and Data Mining, AAAI Press, Menlo Park

Komorowski J.and Zytkow J., 1997, Principles of data mining and knowledge discovery, Springer, New York

Kusiak A., 2001, "Feature transformation methods in data mining," IEEE Transaction on Electronics Packaging Manufacturing, Vol. 24, No. 3, pp.214-21.

Laaksonen, Jorma T., Koskela J. Markus and Oja, Erkki, 2004, "Class distributions on SOM surfaces for feature extraction and object retrieval," Neural Networks, Vol.17, pp.1121-1133.

Lee, Chi Hoon, Shin, Sung Woo and Chung, Jin Wook, 2006, "Network Intrusion Detection Through Genetic Feature Selection," Proceedings of the Seventh ACIS International Conference on Software Engineering, Artificial Intelligence, Networking, and Parallel/Distributed Computing, pp.109.

Liou, James J.H., 2009, "A novel decision rules approach for customer relationship management of the airline market," Expert Systems with Applications, Vol. 36, pp. 4374-4381.

Liou, James J.H., 2011, "Variable Consistency Dominance-based Rough Set Approach to formulate airline service strategies," Applied Soft Computing, Vol. 11, pp. 4011-4020. 
Liou, James J.H., Yen, Leon and Tzeng, Gwo-Hshiung, 2010, "Using decision rules to achieve mass customization of airline services," European Journal of Operational Research, Vol.205, pp.680-686.

Liu, Y., Xu, C. and Pan, Y., 2004, "An incremental rule extracting algorithm based on Pawlak reduction," IEEE International Conference on Systems, Zhejiang, China, pp. 5964-5968.

Lusch, R. F. and Vargo , L., 2006. The Service-Dominant Logic of Marketing: Dialog, Debate, and Directions, M.E. Sharpe

Lynch, C, 2008, "Big data: How do your data grow?" Nature, Vol.7209(455), pp.28-29.

Manyika, J., McKinsey Global Institute, Chui, M., Brown, B., Bughin, J., Dobbs, R., \& Byers, A. H., 2011, "Big data: The next frontier for innovation, competition, and productivity." McKinsey Global Institute.

Nakariyakul, Songyot and Casasent, David P., 2007, "Adaptive branch and bound algorithm for selecting optimal features," Pattern Recognition Letters, Vol.28, pp.1415-1427.

Pawlak, Zdzisław, 1991, Rough Sets: Theoretical Aspects of Reasoning about Data, Kluwer Academic Publishers, Boston.

Qiu, R., Z. Fang, H. Shen, M. Yu., 2007, "Editorial: towards service science, engineering and practice," International Journal of Services Operations and Informatics, Vol. 2, No. 2 pp.103-113

Qiu, Robin G., 2009, “Computational Thinking of Service Systems: Dynamics and Adaptiveness Modeling," Service Science, Vol.1, No.1, pp.42-55.

Saravanan, N., Ramachandran, K.I., 2010, "Incipient gear box fault diagnosis using discrete wavelet transform (DWT) for feature extraction and classification using artificial neural network (ANN)," Expert Systems with Applications, Vol. 37, pp.4168-4181.

Spohrer, J., Maglio, P., Bailey, J. and Gruhl, D., 2007. Steps toward a science of service systems. IEEE Computer Magazine, Vol. 40, Issue. 1, pp. 71-77.

Tseng, Tzu-Liang (Bill), Huang, Chun-Che, 2007, "Rough set-based approach to feature selection in customer relationship management," Omega, Vol.35, pp.365 - 383.

Van Coillie, Frieke M.B., Verbeke, Lieven P.C. and Wulf, Robert R. De, 2007, "Feature selection by genetic algorithms in object-based classification of IKONOS imagery for forest mapping in Flanders, Belgium," Remote Sensing of Environment, Vol. 110, pp.476- 487.

Wang, Jue, Guo, Kun and Wang, Shouyang, 2010, "Rough set and Tabu search based feature selection for credit scoring," Procedia Computer Science, Vol.1, pp.2425-2432. 
Wang, Yong, Li, Lin, Ni, Jun and Huan, Shuhong, 2009, "Feature selection using tabu search with long-term memories and probabilistic neural networks," Pattern Recognition Letters, Vol.30, pp.661-670.

Yu, PS.,1999, "Data mining and personalization technologies," Proceedings of the sixth International Conference on Database Systems for Advanced Applications, Hsinchu, Taiwan, pp. 6-13. 


\section{Acknowledgements}

The authors of this paper would like to thank the National Science Foundation (Grant No: NSF DUE-TUES-1246050) for its financial support towards the project. 